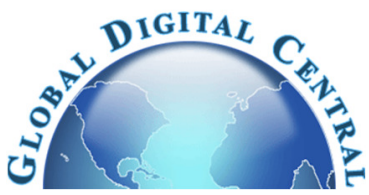

\title{
UNSTEADY BOUNDARY LAYER FLOW AND HEAT TRANSFER OF MAXWELL VISCOELASTIC FLUID WITH TIME FRACTIONAL CATTANEO-CHRISTOV HEAT FLUX MODEL
}

\author{
Mengchen Zhang ${ }^{\mathrm{a}}$, Hui Chen ${ }^{\mathrm{b}, *}$, Ming Shen ${ }^{\mathrm{a}}$ \\ ${ }^{a}$ College of Mathematics and Computer Science, Fuzhou University, Fuzhou 350116, China \\ ${ }^{b}$ School of Mechanical Engineering and Automation, Fuzhou University, Fuzhou 350116, China
}

\begin{abstract}
The time fractional Cattaneo-Christov flux heat model is first introduced to investigate the flow and heat transfer of Maxwell viscoelastic fluid past a vertical flat plate. Fractional constitutive relation and Cattaneo-Christov heat flux model are applied to construct the governing boundary layer equations of momentum and energy, which are nondimensionalized by new dimensionless variables and solved numerically. The results indicate that there exist intersections on velocity and temperature profiles for different values of Prandtl number when the fractional Cattaneo-Christov flux heat model is considered.
\end{abstract}

Keywords: Maxwell viscoelastic fluid; boundary layer flow; time fractional Cattaneo-Christov heat flux model.

\section{INTRODUCTION}

The study on heat transfer has attracted a considerable attention due to its widespread existence in many fields. The Fourier heat flux law (Grattan-Guinness, 2005) has been applied to investigate the features of heat transfer in the last two centuries. However, it leads to the paradox of infinite speed of propagation. In order to overcome this drawback, Cattaneo (2011) proposed a modified Fourier heat conduction law by adding a relaxation time term. So the diffusion equation is turned from a parabolic equation to a hyperbolic one, but the relation only involves partial time derivative. Recently Christov (2009) proposed an extension for the Cattaneo's law by using Oldroyd's upper-convected derivative, which successfully preserves the material-invariant formulation. This Cattaneo-Christov heat flux law is given by

$q+\xi\left[\frac{\partial q}{\partial t}+V \cdot \nabla q-q \cdot \nabla V+(\nabla \cdot V) q\right]=-k \nabla T$

in which $\xi, V, k$ and $T$ represent the relaxation parameter, velocity vector, thermal conductivity and temperature respectively. The Cattaneo-Christov heat flux model has been employed to predict the heat transport behavior under different mechanical and thermal boundary conditions (Straughan, 2010; Waqas et al., 2016; Han et al., 2014; Hayat et al., 2016; Sui et al., 2016). Hayat et al., (2017) have investigated the heat and mass transfer of the boundary-layer flow of Burgers nanofluid with Cattaneo-Christov double diffusion. Also, they have considered the 3D flow of Prandtl liquid by employing CattaneoChristov double diffusion models (Hayat et al., 2018).

Viscoelastic fluids have gained tremendous attention of researchers (Hayat et al., 2016; Hayat et al., 2016; Hayat et al., 2017) due to their wide application in different fields of engineering and industry, such as composite manufacturing process, polymer melts and solutions, tissue engineering and enhanced oil recovery. Constitutive equations with fractional derivatives have long played an important role in the description of complex dynamics in viscoelastic fluids (Song et al., 2000; Tan and Xu, 2002) as the fractional derivative is flexible. The Maxwell fluid is an important class of viscoelastic fluids, and the constitutive relation of Maxwell viscoelastic fluid written in terms of the fractional calculus has been shown to be consistent with thermodynamic principles (Friedrich, 1991), which is introduced as

$$
\left(1+\lambda^{\alpha} \frac{\partial^{\alpha}}{\partial t^{\alpha}}\right) \sigma_{x y}=\mu \frac{\partial u}{\partial y}
$$

where $\lambda$ is the relaxation time of heat conduction, $\alpha(0<\alpha \leq 1)$ is the velocity fractional derivative parameter, $\sigma_{x y}$ is the shear stress component, $\mu$ is the kinematic viscosity, $\partial^{\alpha} / \partial t^{\alpha}$ is the Caputo fractional derivative operator and the fractional derivative of order $\alpha$ is defined as (Podlubny, 1999):

$$
\frac{\partial^{\alpha} \sigma(x, y, t)}{\partial t^{\alpha}}=\frac{1}{\Gamma(1-\alpha)} \int_{0}^{t} \frac{1}{(t-\tau)^{\alpha}} \frac{\partial \sigma(x, y, t)}{\partial \tau} d \tau
$$

where $\Gamma(\cdot)$ is the Gamma function.

The fractional derivative is a global operator reflecting memory character (Du et al.,2012), which has been verified effective in different areas. The study for the application of fractional derivative operator has attracted much interest in recent years (Chen et al., 2013; Jiang and Qi, 2012; Yu et al., 2015). Ghazizadeh et al. (2010) studied the numerical solution of fractional order Cattaneo equation for describing anomalous diffusion. Fetecau et al. (2009) determined the velocity field and the adequate shear stress corresponding to the unsteady flow of a generalized Maxwell fluid by using Fourier sine and Laplace transforms. Tripathi et al. (2010) presents the transportation of viscoelastic fluid with fractional Maxwell model through a channel.

In the study of heat conduction processes, fractional calculus theory has also been applied to anomalous heat conduction owing to the

\footnotetext{
* Correspondence author. Email: felixceng@ @fu.edu.cn
} 
nonlocal nature of fractional operators. Povstenko (2009) formulated the theory of thermal stresses by the generalized Cattaneo-type equations with Caputo time fractional derivatives. Liu et al. (2016) proposed an improved constitutive model in which the space Riesz fractional Cattaneo-Christov model is used to characterize heat conduction phenomena, and then they (Liu et al., 2017a) presented a new time and space fractional Cattaneo-Christov upper-convective derivative flux heat conduction model where the space fractional derivative is characterized by the weight coefficient of forward versus backward transition probability. In the two papers, the velocity is considered as a constant for simplicity so they only dealt with the energy equation and presented the impacts of fractional parameters evolution on heat transfer characteristics.

Motivated by above discussions, we investigate the flow and heat transfer of Maxwell viscoelastic past a vertical flat plate with the time fractional Cattaneo-Christov heat flux, which can be rewritten as (Liu et al., 2017b)

$$
q+\xi\left[\tau^{\beta-1} \frac{\partial^{\beta} q}{\partial t^{\beta}}+(V \cdot \nabla q-q \cdot \nabla V+(\nabla \cdot V) q)\right]=-k \nabla T,
$$

where $\tau$ is introduced to keep the dimension of constitutive equation balance and its dimension is "s", $\beta(0<\beta \leq 1)$ is the velocity fractional derivative parameter, the symbol $\partial^{\beta} / \partial t^{\beta}$ is the Caputo's time fractional derivative of order $\beta$. By the new dimensionless variables, the nonlinear governing equations with mixed time-space derivatives are nondimensionalized and solved numerically. The effects of embedded parameters on velocity and temperature profiles are presented graphically and analyzed in detail.

\section{MATHEMATICAL FORMULATION}

Consider two-dimensional unsteady boundary layer flow and heat transfer of Maxwell viscoelastic fluid past a vertical plate. The Cartesian coordinate system is considered in a way that the $\mathrm{x}$-axis is along the plate and $y$-axis is perpendicular to the plate. It is also presumed that $T_{\mathrm{w}}$ is the temperature of the plate and the ambient temperature corresponds to $T_{\infty}$. The diagram of the physical model is described in Fig. 1.

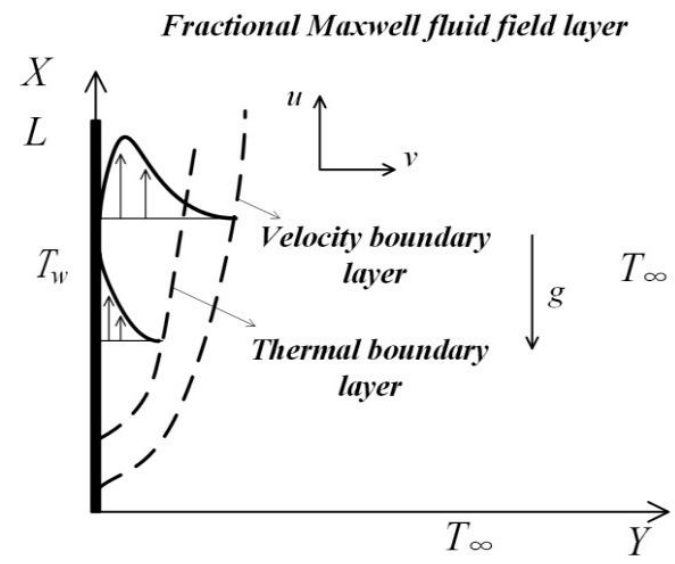

Fig. 1 The Schematic diagram of the physical model

By using the constitutive relation of viscoelastic fluid with fractional Maxwell model (2), the boundary layer equations of continuity and momentum can be written as follows (Zhao, 2016):

$\frac{\partial u}{\partial x}+\frac{\partial v}{\partial y}=0$

$\left(1+\lambda^{\alpha} D_{t}^{\alpha}\right)\left(\frac{\partial u}{\partial t}+u \frac{\partial u}{\partial x}+v \frac{\partial u}{\partial y}\right)=v_{f} \frac{\partial^{2} u}{\partial y^{2}}+g \beta_{T}\left(1+\lambda^{\alpha} D_{t}^{\alpha}\right)\left(T-T_{\infty}\right)$ where $u, v$ and $T$ are the velocity components and temperature respectively, $\rho$ is the density of fluid, $\beta_{\mathrm{T}}$ is the volumetric thermal expansion coefficient. It is worth noting that when $\alpha=0$ the model is simplified as the classical Newtonian fluid, while $\alpha=1$ is corresponding to the ordinary Maxwell model.

Combining time fractional Cattaneo-Christov flux (4) with the following energy conservation equation (Povstenko, 2011)

$c \rho\left(\frac{\partial T}{\partial t}+u \frac{\partial T}{\partial x}+v \frac{\partial T}{\partial y}\right)=-\nabla \cdot q$,

the fractional boundary layer energy equation can be obtained as

$$
\begin{array}{r}
\frac{\partial T}{\partial t}+u \frac{\partial T}{\partial x}+v \frac{\partial T}{\partial y}+\xi \tau^{\beta-1} \frac{\partial^{\beta+1} T}{\partial t^{\beta+1}}+\xi \tau^{\beta-1} u \frac{\partial^{\beta+1} T}{\partial x \partial t^{\beta}}+\xi \tau^{\beta-1} v \frac{\partial^{\beta+1} T}{\partial y \partial t^{\beta}} \\
+\xi r\left(u \frac{\partial^{2} T}{\partial x \partial t}+v \frac{\partial^{2} T}{\partial y \partial t}+u^{2} \frac{\partial^{2} T}{\partial x^{2}}+v^{2} \frac{\partial^{2} T}{\partial y^{2}}+u \frac{\partial u}{\partial x} \frac{\partial T}{\partial x}+u \frac{\partial v}{\partial x} \frac{\partial T}{\partial y}\right. \\
\left.+2 u v \frac{\partial^{2} T}{\partial x \partial y}+v \frac{\partial u}{\partial y} \frac{\partial T}{\partial x}+v \frac{\partial v}{\partial y} \frac{\partial T}{\partial y}\right)=\alpha_{f} \frac{\partial^{2} T}{\partial y^{2}}
\end{array}
$$

where $\alpha_{f}=k / c \rho$ is the thermal diffusion coefficient, $c$ is the specific heat capacity. By setting $\xi=0$, Eq. (8) reduces to the classical heat conduction model.

The initial and boundary conditions are given as follows:

$$
\begin{gathered}
x \geq 0: u(x, y, 0)=0, v(x, y, 0)=0, \mathrm{~T}(x, y, 0)=T_{\infty} ; \\
t>0: u(x, 0, t)=0, v(x, 0, t)=0, \mathrm{~T}(x, 0, t)=T_{w} ; \\
u(0, y, t)=0, \mathrm{~T}(0, y, t)=T_{\infty} ; \\
u(x, y, t) \rightarrow 0, \mathrm{~T}(x, y, t) \rightarrow T_{\infty}, y \rightarrow \infty .
\end{gathered}
$$

In order to simplify the study, the dimensionless variables are introduced as follows:

$$
\begin{gathered}
x^{*}=\frac{L^{3} x}{v_{f}{ }^{2} \tau^{2} \mathrm{Gr}}, y^{*}=\frac{y}{\left(v_{f} \tau\right)^{1 / 2}}, u^{*}=\frac{L^{3} u}{v_{f}{ }^{2} \tau \mathrm{Gr}}, v^{*}=\left(\frac{\tau}{v_{f}}\right)^{1 / 2} v, \\
t^{*}=\frac{t}{\tau}, \theta=\frac{T-T_{\infty}}{T_{w}-T_{\infty}}, \lambda^{*}=\frac{\lambda}{\tau}, \xi^{*}=\frac{\xi}{\tau}, \mathrm{Gr}=\frac{g \beta_{T} L^{3}\left(T_{w}-T_{\infty}\right)}{v_{f}{ }^{2}}, \operatorname{Pr}=\frac{v_{f}}{\alpha_{f}},
\end{gathered}
$$

where $L$ is the length of the vertical plate, Gr and Pr are the Grashof number and Prandtl number respectively. By omitting the dimensionless mart $*$ for simplicity, the dimensionless governing equations can be obtained as below:

$$
\begin{array}{r}
\frac{\partial u}{\partial x}+\frac{\partial v}{\partial y}=0, \\
\left(1+\lambda^{\alpha} D_{t}^{\alpha}\right)\left(\frac{\partial u}{\partial t}+u \frac{\partial u}{\partial x}+v \frac{\partial u}{\partial y}\right)=\frac{\partial^{2} u}{\partial y^{2}}+\left(1+\lambda^{\alpha} D_{t}^{\alpha}\right) \theta \\
\frac{\partial \theta}{\partial t}+u \frac{\partial \theta}{\partial x}+v \frac{\partial \theta}{\partial y}+\xi \frac{\partial^{\beta+1} \theta}{\partial t^{\beta+1}}+\xi u \frac{\partial^{\beta+1} \theta}{\partial x \partial t^{\beta}}+\xi v \frac{\partial^{\beta+1} \theta}{\partial y \partial t^{\beta}}+\xi r\left(u \frac{\partial^{2} \theta}{\partial x \partial t}\right. \\
+v \frac{\partial^{2} \theta}{\partial y \partial t}+u^{2} \frac{\partial^{2} \theta}{\partial x^{2}}+v^{2} \frac{\partial^{2} \theta}{\partial y^{2}}+u \frac{\partial u}{\partial x} \frac{\partial \theta}{\partial x}+u \frac{\partial v}{\partial x} \frac{\partial \theta}{\partial y} \\
\left.+2 u v \frac{\partial^{2} \theta}{\partial x \partial y}+v \frac{\partial u}{\partial y} \frac{\partial \theta}{\partial x}+v \frac{\partial v}{\partial y} \frac{\partial \theta}{\partial y}\right)=\frac{1}{\operatorname{Pr} \frac{\partial^{2} \theta}{\partial y^{2}}}
\end{array}
$$

The corresponding non-dimensional initial and boundary conditions become:

$$
\begin{gathered}
x \geq 0: u(x, y, 0)=0, v(x, y, 0)=0, \theta(x, y, 0)=0 ; \\
t>0: u(x, 0, t)=0, v(x, 0, t)=0, \theta(x, 0, t)=1 ; \\
u(0, y, t)=0, \theta(0, y, t)=0 ; \\
u(x, y, t) \rightarrow 0, \theta(x, y, t) \rightarrow 0, \text { as } y \rightarrow \infty .
\end{gathered}
$$




\section{NUMERICAL TECHNIQUES}

\subsection{Discretization Method}

Define $x_{i}=i \Delta x(i=0,1,2, \ldots, \mathrm{N}), y_{j}=j \Delta y(j=0,1,2, \ldots, \mathrm{M}), t_{k}=k \Delta t(k=0$, $1,2, \ldots, \mathrm{R})$, where $\Delta x=L / N$ and $\Delta y=Y_{\max } / M$ are space step, $\Delta t$ is time step. $u^{\mathrm{k}} \mathrm{i}, \mathrm{j}$ is the numerical solution of equations (10)-(12) at the mesh point $\left(x_{\mathrm{i}}, y_{\mathrm{j}}, t_{\mathrm{k}}\right)$. According to the definition of Caputo fractional derivative, we introduce the L1-algorithm to discretize the time fractional derivative $(0<\beta<1)$ in the following form (Liu et al., 2007):

$$
\begin{aligned}
\frac{\partial^{\beta} f\left(t_{k}\right)}{\partial t^{\beta}} & =\frac{\Delta t^{-\beta}}{\Gamma(2-\beta)} \sum_{q=0}^{k-1} b_{q}\left[f\left(t_{k-q}\right)-f\left(t_{k-q-1}\right)\right]+\mathrm{O}\left(\Delta t^{2-\beta}\right) \\
& =\frac{\Delta t^{-\beta}}{\Gamma(2-\beta)}\left[f\left(t_{k}\right)-b_{k-1} f\left(t_{0}\right)-\sum_{q=1}^{k-1}\left(b_{q-1}-b_{q}\right) f\left(t_{k-q}\right)\right] \\
& +\mathrm{O}\left(\Delta t^{2-\beta}\right),
\end{aligned}
$$

where $b_{q}=(q+1)^{1-\beta}-q^{1-\beta}, q=0,1,2, \ldots, R$. The detailed difference schemes and iterative equations are shown in the appendix.

\subsection{Iteration algorithm}

According to the initial condition, we can acquire the values of $u, v$ and $\theta$ in the specific domain at $t=0$. The variables of $(k-1)$-level are regarded as constants. The iteration equations can be written as tri-diagonal system of equations, then their solutions can be obtained by the Tomas algorithm (Carnahan et al., 1969). The values of (i-1)-level only influence the right side of the linear equations. When the absolute values of the difference between velocity $u$ and temperature $\theta$ at all nodes within two consecutive time steps are less than $10^{-5}$, the iteration of time level stop and arrives at the steady state. Regarding the computational region as a rectangle with boundary of $x_{\max }=1$ and $y_{\max }=12$, where $y_{\max }$ corresponds to $y \rightarrow \infty$. After the consideration of the accuracy of numerical solutions and the time of computation, the spatial mesh sizes and time steps are fixed as $\Delta x=0.05, \Delta y=0.05, \Delta t=0.1$. Based on the computation of the values of $u$ and $\theta$ with the following mesh sizes in Fig. 2, the stability and convergence of the numerical solutions for the selected grid sizes can be proved.

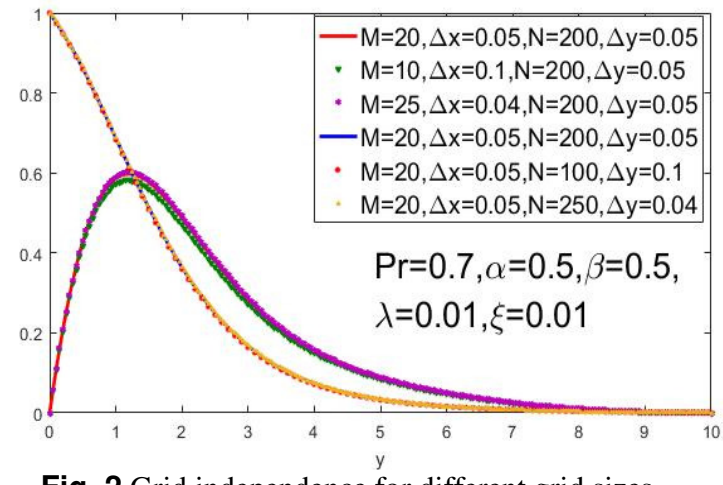

Fig. 2 Grid independence for different grid sizes

Table 1 Comparison between $-\partial \theta /\left.\partial y\right|_{y=0}$ at $x=1$ for $\lambda=0, \xi=0$ and similarity solutions $-\theta^{\prime}(0)$

\begin{tabular}{ccccc}
\hline \hline $\operatorname{Pr}$ & $\begin{array}{c}\text { Crepeau and } \\
\text { Clarksean } \\
(1997)\end{array}$ & $\begin{array}{c}\text { Chamkh and } \\
\text { Khaled } \\
(2001)\end{array}$ & $\begin{array}{c}\text { Chen } \\
(2004)\end{array}$ & $\begin{array}{c}\text { Present } \\
\text { Study }\end{array}$ \\
\hline 0.1 & 0.2302 & 0.2119 & 0.2301 & 0.2249 \\
1 & 0.5671 & 0.5646 & 0.5671 & 0.5705 \\
10 & 1.1690 & 1.1720 & 1.1693 & 1.1758 \\
\hline \hline
\end{tabular}

In order to verify the accuracy of the numerical solutions, a comparison between $-\partial \theta /\left.\partial y\right|_{y=0}$ at $x=1$ when $\lambda=0, \xi=0$ and the previous published similarity solutions $-\theta^{\prime}(0)$ (Crepeau and Clarksean, 1997; Chamkha and Khaled, 2001; Chen, 2004) are demonstrated in Table 1.
The results indicate that the numerical solutions in the paper are consistent with the previous published data.

\section{RESULTS AND DISCUSSION}

The present section aims to study the impacts of fractional derivative parameters $\alpha$ and $\beta$ on the dimensionless velocity $u$ and temperature $\theta$. Moreover, we show the influence of Pr on velocity and temperature at both cases of classical heat flux $\xi=0$ and fractional Cattaneo-Christov heat flux model.

Figures. 3 and 4 present the effects of fractional parameters $\alpha$ and $\beta$ on the velocity distributions. It is shown from Fig. 3 that the maximum value of velocity profile reduces with $\alpha$ while the position of maximum value gets closer to the vertical plate. The thickness of the momentum boundary layer increases with $\alpha$ slightly, which indicates that the velocity fractional derivative parameter weakens the natural convection flow and boosts the elastic effect of Maxwell fluid. It is also worth noting that the velocity profiles intersect each other for different values of $\alpha$, which implies that the fractional equation with relaxation times shows short-term memory for the previous moment and try to go back to the previous state. Unlike the influence of $\alpha$ on velocity, the maximum values of velocity remain mostly unchanged with different values of $\beta$ as show in Fig. 4. The temperature fractional derivative parameter $\beta$ has almost no influence on the velocity profiles near the vertical plate. However, the velocity increases remarkably with the increase of $\beta$.

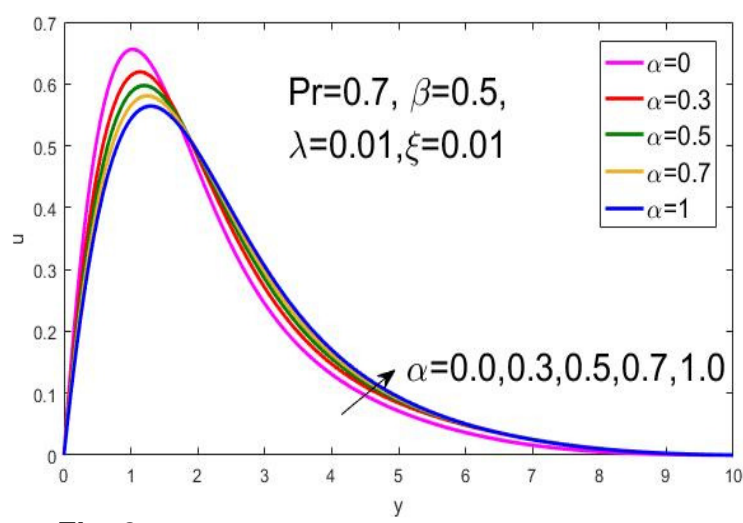

Fig. 3 Velocity profiles for different $\alpha$

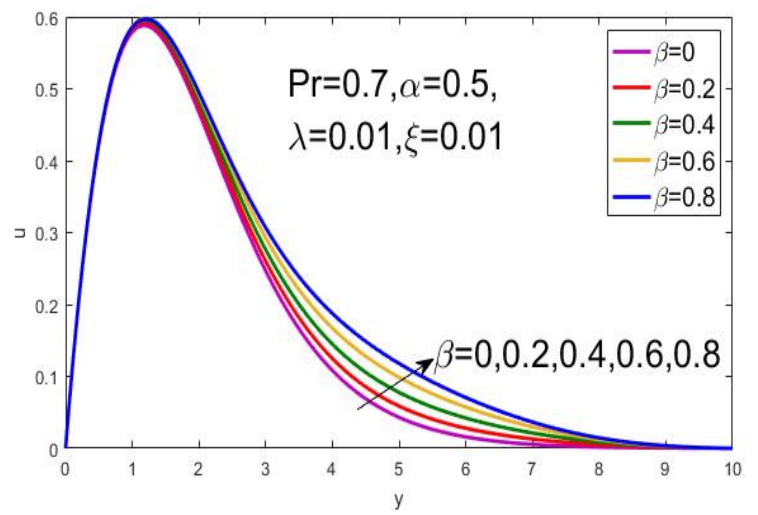

Fig. 4 Velocity profiles for different $\beta$

The influences of $\alpha$ and $\beta$ on the temperature distributions are illustrated in Figs. 5 and 6. It is shown in Fig. 5 that the temperature profiles rise with the increase of $\alpha$. It is observed that the classical Newtonian fluid has the thinnest boundary layer, while the ordinary Maxwell fluid is corresponding to the thickest thermal boundary layer. The temperature distribution rises and the thermal boundary layer is thicker for larger values of $\alpha$. On the contrary, the temperature profiles 
decline with the increase of $\beta$ as depicted in Fig. 6, which demonstrate a loss of the thickness of thermal boundary layer. These results indicate that the temperature frictional parameter $\beta$ enhances the efficiency of heat transfer.

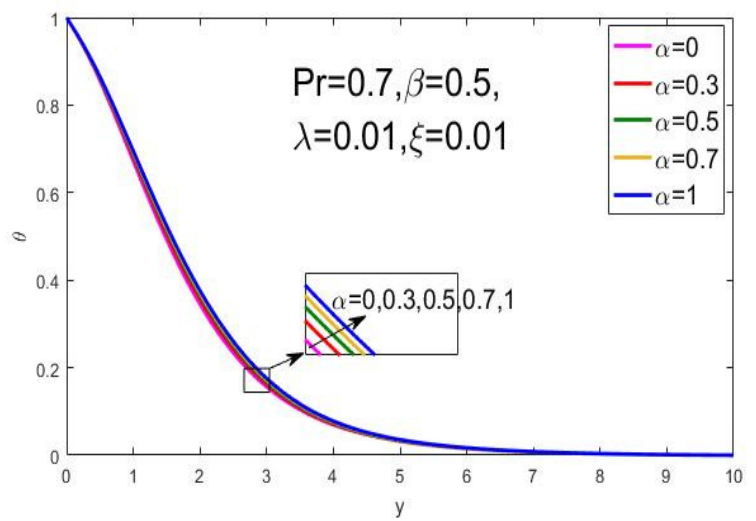

Fig. 5 Temperature profiles for different $\alpha$

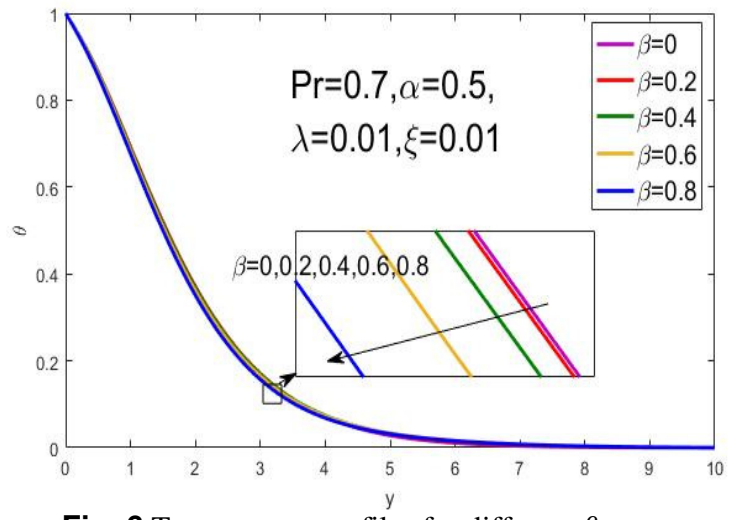

Fig. 6 Temperature profiles for different $\beta$

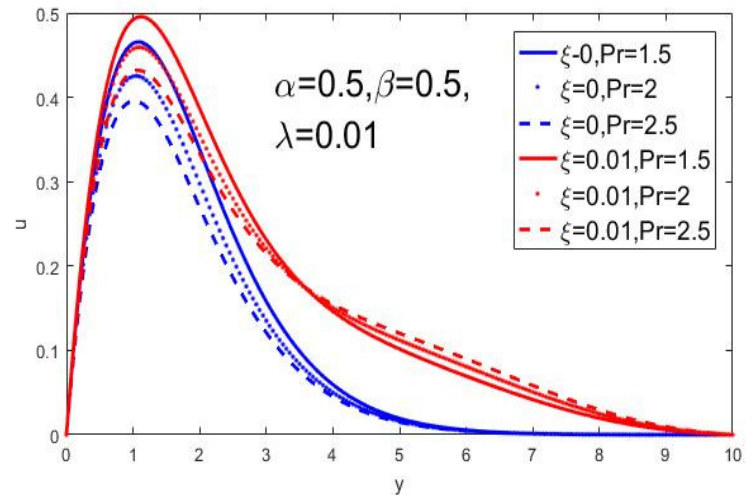

Fig. 7 Velocity profiles of two different models for different $\mathrm{Pr}$

The comparisons of velocity and temperature distributions between the classical heat flux model and the frictional CattaneoChristov heat flux model are shown in Figs. 7 and 8, respectively. The frictional Cattaneo-Christov heat flux model reduces to the classical heat flux model when the relaxation parameter $\xi=0$. It is observed from Fig. 7 that the maximum value of velocity and the thickness of momentum boundary layer for the fractional Cattaneo-Christov model are larger than them for the classical heat flux model when the Prandtl number is fixed. Moreover, with the increasing of Prandtl number, the value of maximum velocity declines and goes left position slightly to the vertical plate for the two cases. It is indicated from Fig. 8 that for every fixed Prandtl number, the thickness of thermal boundary layer for the Cattaneo-Christov model is larger compared to the classical heat flux model. Furthermore, it is worth noting that the velocity profiles as well as the temperature profiles intersect each other for the case of the fractional Cattaneo-Christov model. The existence of intersections implies that the frictional Cattaneo-Christov heat flux model is able to describe the influence of memory on the viscoelastic fluid behavior.

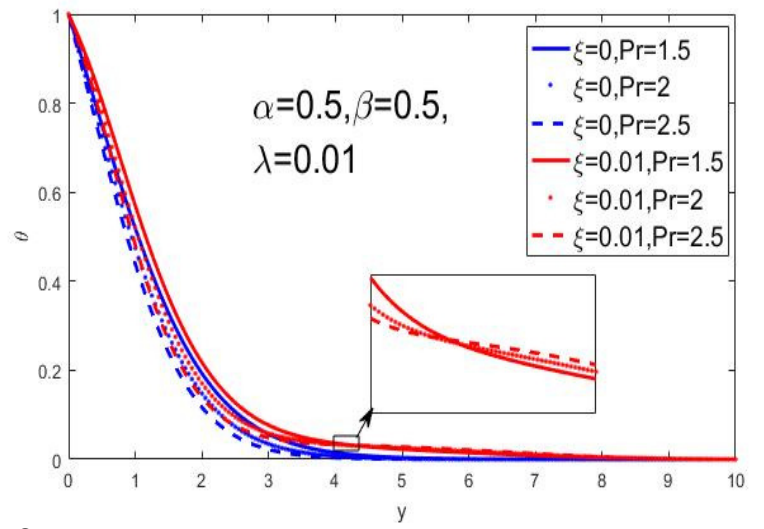

Fig. 8 Temperature profiles of two different models for different $\mathrm{Pr}$

\section{CONCLUSIONS}

The paper investigates unsteady flow of Maxwell viscoelastic fluid with time fractional Cattaneo-Christov heat flux model. The fractional Maxwell shear stress and time fractional Cattaneo-Christov heat flux models are introduced in the constitutive relations. The new dimensionless variables are proposed to nondimensionalize the governing equations, which are solved by finite difference method together with L1-algorithm. The important observations of the present study are summarized as follows:

- The velocity fractional derivative parameter $\alpha$ weakens the natural convection flow and enhances the elastic effect of Maxwell fluid.

- The temperature declines and the thermal boundary layer becomes thinner as $\beta$ increase, which implies that the temperature fractional parameter enhances the efficiency of heat transfer

- The influence of fractional derivative parameter $\alpha$ on temperature distributions is opposite to $\beta$.

- The velocity and temperature profiles intersect each other for different values of Prandtl number for the case of time fractional Cattaneo-Christov heat flux model.

- The fractional Cattaneo-Christov heat flux model with Caputo time derivatives exhibits a short memory of previous states.

\section{ACKNOWLEDGEMENTS}

This work is supported by Collaborative Innovation Center of High-end Equipment Manufacturing in Fujian and the National Natural Science Foundation of China (Grant No. 51305080).

\section{NOMENCLATURE}

c specific heat capacity $(\mathrm{J} / \mathrm{kg} \cdot \mathrm{K})$

Gr Grashof number

$g \quad$ acceleration due to gravity $\left(\mathrm{m} / \mathrm{s}^{2}\right)$

$k \quad$ thermal conductivity $(\mathrm{W} / \mathrm{m} \cdot \mathrm{K})$

$L \quad$ length of the vertical plate (m)

Pr Prandtl number

$q \quad$ heat flux $\left(\mathrm{W} / \mathrm{m}^{2}\right)$

$T \quad$ temperature $(\mathrm{K})$

time (s)

interfacial velocity component $(\mathrm{m} / \mathrm{s})$

interfacial velocity component $(\mathrm{m} / \mathrm{s})$ 
$x \quad$ coordinate component $(\mathrm{m})$

$y \quad$ coordinate component $(\mathrm{m})$

\section{Greek Symbols}

$\alpha \quad$ velocity fractional derivative parameter

$\alpha_{f} \quad$ thermal diffusion coefficient $\left(\mathrm{m}^{2} / \mathrm{s}\right)$

$\beta \quad$ time fractional derivative parameter

$\beta_{T} \quad$ volumetric thermal expansion coefficient

$\lambda$ relaxation time of heat conduction

$\mu \quad$ dynamic viscosity $\left(\mathrm{m}^{2} / \mathrm{s}\right)$

$\theta \quad$ dimensionless temperature

$\rho \quad$ density $\left(\mathrm{kg} / \mathrm{m}^{3}\right)$

$\sigma_{x y} \quad$ shear stress component (pa)

$\tau \quad$ the coefficient to keep the dimension of constitutive equation balance (s)

vf kinematic viscosity $\left(\mathrm{m}^{2} / \mathrm{s}\right)$

$\xi \quad$ relaxation parameter

Superscripts

* dimensionless form

Subscripts

$t \quad$ time derivative

$w \quad$ vertical plate

$\infty \quad$ ambient environment

\section{REFERENCES}

Carnahan, B., Luther, H.A., and Wilkes, J. O., 1969, "Applied Numerical Methods," John Wiley \& Sons, New York. https://doi.org/10.1002/nme.1620040415

Cattaneo, C., 2011, "Sulla Conduzione Del Calore. Some Aspects of Diffusion Theory," Springer Berlin Heidelberg, 83-101.

https://doi.org/10.1007/978-3-642-11051-1_5

Chamkha A.J., Khaled A.R.A., 2001, "Similarity solutions for hydromagnetic simultaneous heat and mass transfer by natural convection from an inclined plate with internal heat generation or absorption," Heat \& Mass Transfer, 37(2-3), 117-123.

https://doi.org/10.1007/s002310000131

Chen C.H., 2004, "Combined heat and mass transfer in MHD free convection from a vertical surface with Ohmic heating and viscous dissipation," International Journal of Engineering Science, 42(7), 699713.

https://doi.org/10.1016/j.ijengsci.2003.09.002

Chen W., Zhang J., Zhang J., 2013, “A variable-order time-fractional derivative model for chloride ions sub-diffusion in concrete structures," Fractional Calculus \& Applied Analysis, 16(1), 76-92.

https://doi.org/10.2478/s13540-013-0006-y

Christov C.I., 2009, "On frame indifferent formulation of the MaxwellCattaneo model of finite-speed heat conduction," Mechanics Research Communications, 36(4), 481-486.

https://doi.org/10.1016/j.mechrescom.2008.11.003

Crepeau J.C., Clarksean R.,1997, "Similarity solutions of natural convection with internal heat generation," Journal of Heat Transfer, 119(1), 183-185.

https://doi.org/10.1115/1.2824086

Du M., Wang Z., Hu H., 2012, "Measuring memory with the order of fractional derivative," Scientific Reports, 3(7478), 3431.

https://doi.org/10.1038/srep03431

Fetecau C., Athar M., Fetecau C., 2009, “Unsteady flow of generalized Maxwell fluid with fractional derivative due to a constantly accelerating plate," Computers \& Mathematics with Applications, 57(4), 596-603. https://doi.org/10.1016/j.camwa.2008.09.052
Friedrich C., 1991, "Relaxation and retardation functions of the Maxwell model with fractional derivatives," Rheologica Acta, 30(2), 151-158. https://doi.org/10.1007/BF01134604

Ghazizadeh H.R., Maerefat M., Azimi A., 2010, "Explicit and implicit finite difference schemes for fractional Cattaneo equation," Journal of Computational Physics, 229(19), 7042-7057.

https://doi.org/10.1016/j.jcp.2010.05.039

Grattan-Guinness I., 2005, "Chapter 26-Joseph Fourier, Théorie analytique de la chaleur, (1822)," Landmark Writings in Western Mathematics 1640-1940, 354-365.

https://doi.org/10.1016/B978-044450871-3/50107-8

Han S., Zheng L., Li C., et al., 2014, "Coupled flow and heat transfer in viscoelastic fluid with Cattaneo-Christov heat flux model," Applied Mathematics Letters, 38, 87-93. https://doi.org/10.1016/j.aml.2014.07.013

Hayat T., Imtiaz M., Alsaedi A., et al., 2016, "On Cattaneo-Christov heat flux in MHD flow of Oldroyd-B fluid with homogeneousheterogeneous reactions," Journal of Magnetism \& Magnetic Materials, 401, 296-303.

https://doi.org/10.1016/j.jmmm.2015.10.039

Hayat, T., Aziz, A., Muhammad, T., and Ahmad, B., 2016, "On magnetohydrodynamic flow of second grade nanofluid over a nonlinear stretching sheet, " Journal of Magnetism \& Magnetic Materials, 408(5), 99-106.

https://doi.org/10.1016/j.jmmm.2016.02.017

Hayat, T., Aziz, A., Muhammad, T., Alsaedi, A., and Mustafa, M., 2016, "On magnetohydrodynamic flow of second grade nanofluid over a convectively heated nonlinear stretching surface, " Advanced Powder Technology, 27(5), 1992-2004. https://doi.org/10.1016/j.apt.2016.07.002

Hayat, T., Aziz, A., Muhammad, T., and Alsaedi, A., 2017, "Active and passive controls of jeffrey nanofluid flow over a nonlinear stretching surface, " Results in Physics, 7, 4071-4078.

https://doi.org/10.1016/j.rinp.2017.10.028

Hayat, T., Aziz, A., Muhammad, T., and Alsaedi, A., 2017, "On model for flow of burgers nanofluid with cattaneo-christov double diffusion, " Chinese Journal of Physics, 55(3), 916-929. https://doi.org/10.1016/j.cjph.2017.02.017

Hayat, T., Aziz, A., Muhammad, T., and Alsaedi, A., 2018, "Threedimensional flow of prandtl fluid with cattaneo-christov double diffusion, " Results in Physics, 9, 290-296.

https://doi.org/10.1016/j.rinp.2018.02.065

Jiang X., Qi H., 2012, "Thermal wave model of bioheat transfer with modified Riemann-Liouville fractional derivative," Journal of Physics A: Mathematical \& Theoretical, 45(48), 485101. https://doi.org/10.1088/1751-8113/45/48/485101

Liu F., Zhuang P., Anh V., et al., 2007, "Stability and convergence next term of the difference methods for the space-time fractional advectiondiffusion equation," Applied Mathematics \& Computation, 191(1), 12 20.

https://doi.org/10.1016/j.amc.2006.08.162

Liu L., Zheng L., Liu F., et al., 2016, "An improved heat conduction model with Riesz fractional Cattaneo-Christov flux," International Journal of Heat \& Mass Transfer, 103, 1191-1197. https://doi.org/10.1016/j.ijheatmasstransfer.2016.07.113

Liu L., Zheng L., Liu F., et al., 2017a, "Heat conduction with fractional Cattaneo-Christov upper-convective derivative flux model," International Journal of Thermal Sciences, 112, 421-426.

https://doi.org/10.1016/j.ijthermalsci.2016.11.008 
Liu L., Zheng L., Liu F., 2017b, "Time fractional Cattaneo-Christov anomalous diffusion in comb frame with finite length of fingers," Journal of Molecular Liquids, 233, 326-333 https://doi.org/10.1016/j.molliq.2017.03.034

Podlubny I., 1999, "Fractional differential equation," Academic Press, San Diego, 78-85.

Povstenko Y.Z., 2009, "Theory of thermoelasticity based on the spacetime-fractional heat conduction equation," Physica Scripta, 2009(T136), 014017.

https://doi.org/10.1088/0031-8949/2009/T136/014017

Povstenko Y.Z., 2011, "Fractional cattaneo-type equations and generalized thermoelasticity," Journal of Thermal Stresses, 34(2), 97114.

https://doi.org/10.1080/01495739.2010.511931

Song D., Song X., Jiang T.Q., et al., 2000, "Study of rheological characterization off Fenugreek gum with modified Maxwell model," Chinese Journal of Chemical Engineering, 8(1), 85-88.

Straughan B., 2010, "Thermal convection with the Cattaneo-Christov model," International Journal of Heat \& Mass Transfer, 53(1-3), 95-98. https://doi.org/10.1016/j.ijheatmasstransfer.2009.10.001

Sui J., Zheng L., Zhang X., 2016, "Boundary layer heat and mass transfer with Cattaneo-Christov double-diffusion in upper-convected Maxwell nanofluid past a stretching sheet with slip velocity," International Journal of Thermal Sciences, 104, 461-468. https://doi.org/10.1016/j.ijthermalsci.2016.02.007

Tan W., Xu M., 2002, "Plane surface suddenly set in motion in a viscoelastic fluid with fractional Maxwell model," Acta Mechanica Sinica, 18(4), 342-349.

https://doi.org/10.1007/BF02487786

Tripathi D., Pandey S.K., Das S., 2010, "Peristaltic flow of viscoelastic fluid with fractional Maxwell model through a channel," Applied Mathematics \& Computation, 215(10), 3645-3654.

https://doi.org/10.1016/j.amc.2009.11.002

Waqas M., Hayat T., Farooq M., et al., 2016, "Cattaneo-Christov heat flux model for flow of variable thermal conductivity generalized Burgers fluid," Journal of Molecular Liquids, 220, 642-648. https://doi.org/10.1016/j.molliq.2016.04.086

Yu B., Jiang X., Xu H., 2015, “A novel compact numerical method for solving the two dimensional non-linear fractional reaction-subdiffusion equation," Numerical Algorithms, 68, 923-950.

https://doi.org/10.1007/s11075-014-9877-1

Zhao, J., Zheng, L., Zhang, X., and Liu, F., 2016, "Unsteady natural convection boundary layer heat transfer of fractional Maxwell viscoelastic fluid over a vertical plate," International Journal of Heat \& Mass Transfer, 97, 760-766.

https://doi.org/10.1016/j.ijheatmasstransfer.2016.02.059

\section{APPENDIX}

By using the backward and central difference, the integer-order terms in the governing equations are discretized as

$\frac{\partial^{\beta}}{\partial t^{\beta}}\left(u \frac{\partial \theta}{\partial x}\right)=\frac{\Delta t^{-\beta}}{\Delta x \Gamma(2-\beta)}\left[\begin{array}{l}\theta_{i, j}^{k-1}\left(\theta_{i, j}^{k}-\theta_{i-1, j}^{k}\right) \\ -\sum_{q=1}^{k-1}\left(b_{q-1}-b_{q}\right) u_{i, j}^{k-q-1}\left(\theta_{i, j}^{k-q}-\theta_{i-1, j}^{k-q}\right)\end{array}\right]$,

where the truncation error is $\mathrm{O}\left(\Delta t^{2-\beta}+\Delta x\right)$.

$$
\frac{\partial^{\beta}}{\partial t^{\beta}}\left(v \frac{\partial \theta}{\partial y}\right)=\frac{\Delta t^{-\beta}}{\Delta y \Gamma(2-\beta)}\left[\begin{array}{l}
\theta_{i, j}^{k-1}\left(\theta_{i, j}^{k}-\theta_{i, j-1}^{k}\right) \\
-\sum_{q=1}^{k-1}\left(b_{q-1}-b_{q}\right) v_{i, j}^{k-q-1}\left(\theta_{i, j}^{k-q}-\theta_{i, j-1}^{k-q}\right)
\end{array}\right],
$$

$\left.\frac{\partial \theta}{\partial t}\right|_{t=t_{k}}=\frac{\theta\left(x_{i}, y_{j}, t_{k}\right)-\theta\left(x_{i}, y_{j}, t_{k-1}\right)}{\Delta t}+\mathrm{O}(\Delta t)$,

$\left.u \frac{\partial \theta}{\partial x}\right|_{t=t_{k}}=u\left(x_{i}, y_{j}, t_{k-1}\right) \frac{\theta\left(x_{i}, y_{j}, t_{k}\right)-\theta\left(x_{i-1}, y_{j}, t_{k}\right)}{\Delta x}+\mathrm{O}(\Delta x)$,

$\left.v \frac{\partial \theta}{\partial y}\right|_{t=t_{k}}=v\left(x_{i}, y_{j}, t_{k-1}\right) \frac{\theta\left(x_{i}, y_{j}, t_{k}\right)-\theta\left(x_{i}, y_{j-1}, t_{k}\right)}{\Delta y}+\mathrm{O}(\Delta y)$,

$\left.\frac{\partial^{2} \theta}{\partial y^{2}}\right|_{t=t_{k}}=\frac{\theta\left(x_{i}, y_{j+1}, t_{k}\right)-2 \theta\left(x_{i}, y_{j}, t_{k}\right)+\theta\left(x_{i}, y_{j-1}, t_{k}\right)}{\Delta y^{2}}+\mathrm{O}\left(\Delta y^{2}\right)$,

$\left.u \frac{\partial^{2} \theta}{\partial x \partial t}\right|_{t=t_{k}}=u\left(x_{i}, y_{j}, t_{k-1}\right) \frac{\theta\left(x_{i}, y_{j}, t_{k}\right)+\theta\left(x_{i-1}, y_{j}, t_{k-1}\right)-\theta\left(x_{i-1}, y_{j}, t_{k}\right)-\theta\left(x_{i}, y_{j}, t_{k-1}\right)}{\Delta x \Delta t}$ $+\mathrm{O}(\Delta x \Delta t)$

$\left.v \frac{\partial^{2} \theta}{\partial y \partial t}\right|_{t=t_{k}}=v\left(x_{i}, y_{j}, t_{k-1}\right) \frac{\theta\left(x_{i}, y_{j}, t_{k}\right)+\theta\left(x_{i}, y_{j-1}, t_{k-1}\right)-\theta\left(x_{i}, y_{j-1}, t_{k}\right)-\theta\left(x_{i}, y_{j}, t_{k-1}\right)}{\Delta y \Delta t}$ $+\mathrm{O}(\Delta y \Delta t)$

$\left.u^{2} \frac{\partial^{2} \theta}{\partial x^{2}}\right|_{t=t_{k}}=u^{2}\left(x_{i}, y_{j}, t_{k}\right) \frac{\theta\left(x_{i+1}, y_{j}, t_{k-1}\right)-2 \theta\left(x_{i}, y_{j}, t_{k-1}\right)+\theta\left(x_{i-1}, y_{j}, t_{k-1}\right)}{\Delta x^{2}}$ $+\mathrm{O}\left(\Delta x^{2}\right)$

$\left.v^{2} \frac{\partial^{2} \theta}{\partial y^{2}}\right|_{t=t_{k}}=v^{2}\left(x_{i}, y_{j}, t_{k-1}\right) \frac{\theta\left(x_{i}, y_{j+1}, t_{k}\right)-2 \theta\left(x_{i}, y_{j}, t_{k}\right)+\theta\left(x_{i}, y_{j-1}, t_{k}\right)}{\Delta y^{2}}$ $+\mathrm{O}\left(\Delta y^{2}\right)$

$\left.u \frac{\partial u}{\partial x} \frac{\partial \theta}{\partial x}\right|_{t=t_{k}}=u\left(x_{i}, y_{j}, t_{k-1}\right) \frac{\left(u\left(x_{i}, y_{j}, t_{k}\right)-u\left(x_{i-1}, y_{j}, t_{k}\right)\right)\left(\theta\left(x_{i}, y_{j}, t_{k}\right)-\theta\left(x_{i-1}, y_{j}, t_{k}\right)\right)}{\Delta x^{2}}$ $+\mathrm{O}\left(\Delta x^{2}\right)$

$\left.u \frac{\partial v}{\partial x} \frac{\partial \theta}{\partial y}\right|_{t=t_{k}}=u\left(x_{i}, y_{j}, t_{k-1}\right) \frac{\left(v\left(x_{i}, y_{j}, t_{k}\right)-v\left(x_{i-1}, y_{j}, t_{k}\right)\right)\left(\theta\left(x_{i}, y_{j}, t_{k}\right)-\theta\left(x_{i}, y_{j-1}, t_{k}\right)\right)}{\Delta x \Delta y}$ $+\mathrm{O}(\Delta x \Delta y)$

$\left.u v \frac{\partial^{2} \theta}{\partial x \partial y}\right|_{t=t_{k}}=u\left(x_{i}, y_{j}, t_{k-1}\right) v\left(x_{i}, y_{j}, t_{k-1}\right) \frac{\theta\left(x_{i}, y_{j}, t_{k}\right)+\theta\left(x_{i-1}, y_{j-1}, t_{k}\right)-\theta\left(x_{i-1}, y_{j}, t_{k}\right)-\theta\left(x_{i}, y_{j-1}, t_{k}\right)}{\Delta x \Delta y}$ $+\mathrm{O}(\Delta x \Delta y)$

$\left.v \frac{\partial u}{\partial y} \frac{\partial \theta}{\partial x}\right|_{t=t_{k}}=v\left(x_{i}, y_{j}, t_{k-1}\right) \frac{\left(u\left(x_{i}, y_{j}, t_{k}\right)-u\left(x_{i}, y_{j-1}, t_{k}\right)\right)\left(\theta\left(x_{i}, y_{j}, t_{k}\right)-\theta\left(x_{i-1}, y_{j}, t_{k}\right)\right)}{\Delta x \Delta y}$ $+\mathrm{O}(\Delta x \Delta y)$,

$\left.v \frac{\partial v}{\partial y} \frac{\partial \theta}{\partial y}\right|_{t=t_{k}}=v\left(x_{i}, y_{j}, t_{k-1}\right) \frac{\left(v\left(x_{i}, y_{j}, t_{k}\right)-v\left(x_{i}, y_{j-1}, t_{k}\right)\right)\left(\theta\left(x_{i}, y_{j}, t_{k}\right)-\theta\left(x_{i}, y_{j-1}, t_{k}\right)\right)}{\Delta y^{2}}$ $+\mathrm{O}\left(\Delta y^{2}\right)$

Substituting Eqs. (A1)-(A3) into Eq. (13), the fractional derivatives become:

$$
\frac{\partial^{\beta+1} \theta_{i, j}^{k}}{\partial t^{\beta+1}}=\frac{\Delta t^{-1-\beta}}{\Gamma(2-\beta)}\left[\theta_{i, j}^{k}-\theta_{i, j}^{k-1}-\sum_{q=1}^{k-1}\left(b_{q-1}-b_{q}\right)\left(\theta_{i, j}^{k-q}-\theta_{i, j}^{k-q-1}\right)\right],
$$

where the truncation error is $\mathrm{O}\left(\Delta t^{2-\beta}+\Delta y\right)$.

Similarly, the finite difference method is employed in dimensionless velocity terms. For simplicity, note

$$
\begin{aligned}
& m_{1}=\frac{\lambda^{\alpha} \Delta t^{-\alpha}}{\Gamma(2-\alpha)}, m_{2}=\frac{\Delta t}{\Delta y^{2}}, m_{3}=\frac{\Delta t^{-\beta}}{\Gamma(2-\beta)}, m_{4}=\frac{\Delta t}{\operatorname{Pr} \Delta y^{2}}, \\
& A_{1}=\sum_{s=1}^{k-1}\left(\alpha_{s-1}-\alpha_{s}\right)\left(u_{i, j}^{k-s}-u_{i, j}^{k-s-1}\right), \\
& A_{2}=\sum_{s=1}^{k-1}\left(\alpha_{s-1}-\alpha_{s}\right) u_{i, j}^{k-s-1}\left(u_{i, j}^{k-s}-u_{i-1, j}^{k-s}\right),
\end{aligned}
$$




$$
\begin{aligned}
& A_{3}=\sum_{s=1}^{k-1}\left(\alpha_{s-1}-\alpha_{s}\right) v_{i, j}^{k-s-1}\left(u_{i, j}^{k-s}-u_{i, j-1}^{k-s}\right), \\
& A_{4}=\sum_{s=1}^{k-1}\left(\alpha_{s-1}-\alpha_{s}\right)\left(\theta_{i, j}^{k-s}-\theta_{i, j}^{k-s-1}\right), \\
& C_{1}=\sum_{q=1}^{k-1}\left(b_{q-1}-b_{q}\right)\left(\theta_{i, j}^{k-q}-\theta_{i, j}^{k-q-1}\right), \\
& C_{2}=\sum_{q=1}^{k-1}\left(b_{q-1}-b_{q}\right) u_{i, j}^{k-q-1}\left(\theta_{i, j}^{k-q}-\theta_{i-1, j}^{k-q}\right), \\
& C_{3}=\sum_{q=1}^{k-1}\left(b_{q-1}-b_{q}\right) v_{i, j}^{k-q-1}\left(\theta_{i, j}^{k-q}-\theta_{i, j-1}^{k-q}\right),
\end{aligned}
$$

where $\alpha_{\mathrm{s}}=(\mathrm{s}+1)^{1-\alpha}-\mathrm{s}^{1-\alpha}, s=0,1,2, \ldots, R$.

The iteration equations are achieved in the following forms:

$v_{i, j}^{k}=v_{i, j-1}^{k}-\frac{\Delta y}{\Delta x}\left(u_{i, j}^{k}-u_{i-1, j}^{k}\right)$,

$-\left(1+m_{1}\right) \frac{\Delta t}{\Delta x} u_{i, j}^{k-1} u_{i-1, j}^{k}-\left[\left(1+m_{1}\right) \frac{\Delta t}{\Delta y} v_{i, j}^{k-1}+m_{2}\right] u_{i, j-1}^{k}$

$+\left[\left(1+m_{1}\right)\left(1+\frac{\Delta t}{\Delta x} u_{i, j}^{k-1}\right)+\left(1+m_{1}\right) \frac{\Delta t}{\Delta y} v_{i, j}^{k-1}+2 m_{2}\right] u_{i, j}^{k}-m_{2} u_{i, j+1}^{k}$

$=\left(1+m_{1}\right) u_{i, j}^{k-1}+m_{1}\left(A_{1}+\frac{\Delta t}{\Delta x} A_{2}+\frac{\Delta t}{\Delta y} A_{3}\right)+\frac{\Delta t m_{1}}{2}\left(\theta_{i, j}^{k}+\theta_{i, j}^{k-1}-A_{4}\right)$

$+\frac{\Delta t}{2}\left(\theta_{i, j}^{k}+\theta_{i, j}^{k-1}\right)$

$$
\begin{aligned}
& -\left(1+\xi m_{3}\right) \frac{\Delta t}{\Delta x} u_{i, j}^{k-1} \theta_{i-1, j}^{k}-r \xi\left[\begin{array}{l}
\frac{u_{i, j}^{k-1}}{\Delta x}-\frac{\Delta t}{\Delta x^{2}} u_{i, j}^{k-1}\left(u_{i, j}^{k}-u_{i-1, j}^{k}\right) \\
+\frac{\Delta t}{\Delta x \Delta y} v_{i, j}^{k-1}\left(2 u_{i, j}^{k-1}+u_{i, j}^{k}-u_{i, j-1}^{k}\right)
\end{array}\right] \theta_{i-1, j}^{k} \\
& -\left[\left(1+\xi m_{3}\right) \frac{\Delta t}{\Delta y} v_{i, j}^{k-1}+m_{4}\right] \theta_{i, j-1}^{k}-r \xi\left[\begin{array}{l}
\frac{v_{i, j}^{k-1}}{\Delta x}+\frac{\Delta t}{\Delta y^{2}} v_{i, j}^{k-1}\left(v_{i, j}^{k}-v_{i, j-1}^{k}-v_{i, j}^{k-1}\right) \\
+\frac{\Delta t}{\Delta x \Delta y} u_{i, j}^{k-1}\left(v_{i, j}^{k}-v_{i-1, j}^{k}+2 v_{i, j}^{k-1}\right)
\end{array}\right] \theta_{i, j-1}^{k} \\
& +\left[\begin{array}{l}
\left(1+\xi m_{3}\right)\left(1+\frac{\Delta t}{\Delta x} u_{i, j}^{k-1}\right) \\
+\left(1+\xi m_{3}\right) \frac{\Delta t}{\Delta y} v_{i, j}^{k-1}+2 m_{4}
\end{array}\right] \theta_{i, j}^{k}+r \xi\left[\begin{array}{l}
\frac{u_{i, j}^{k-1}}{\Delta x}+\frac{v_{i, j}^{k-1}}{\Delta y} \\
+\frac{\Delta t}{\Delta y^{2}} v_{i, j}^{k-1}\left(v_{i, j}^{k}-v_{i, j-1}^{k}-2 v_{i, j}^{k-1}\right) \\
+\frac{\Delta t}{\Delta x^{2}} u_{i, j}^{k-1}\left(u_{i, j}^{k}-u_{i-1, j}^{k}\right) \\
+\frac{\Delta t}{\Delta x \Delta y} v_{i, j}^{k-1}\left(u_{i, j}^{k}-u_{i, j-1}^{k}\right) \\
+\frac{\Delta t}{\Delta x \Delta y} u_{i, j}^{k-1}\left(v_{i, j}^{k}-v_{i-1, j}^{k}+2 v_{i, j}^{k-1}\right)
\end{array}\right] \theta_{i, j}^{k} \\
& +\left[\frac{\Delta t}{\Delta y^{2}} r \xi\left(v_{i, j}^{k-1}\right)^{2}-m_{4}\right] \theta_{i, j+1}^{k}+\frac{2 \Delta t}{\Delta x \Delta y} r \xi u_{i, j}^{k-1} v_{i, j}^{k-1} \theta_{i-1, j-1}^{k} \\
& =-r \xi\left[\frac{u_{i, j}^{k-1}}{\Delta x}+\frac{\Delta t}{\Delta x^{2}}\left(u_{i, j}^{k}\right)^{2}\right] \theta_{i-1, j}^{k-1}-r \xi \frac{v_{i, j}^{k-1}}{\Delta y} \theta_{i, j-1}^{k-1}+\left(1+\xi m_{3}\right) \theta_{i, j}^{k-1} \\
& -r \xi \frac{\Delta t}{\Delta x^{2}}\left(u_{i, j}^{k}\right)^{2} \theta_{i+1, j}^{k-1}+r \xi\left[\frac{u_{i, j}^{k-1}}{\Delta x}+\frac{v_{i, j}^{k-1}}{\Delta y}+\frac{2 \Delta t}{\Delta x^{2}}\left(u_{i, j}^{k}\right)^{2}\right] \theta_{i, j}^{k-1} \\
& +\xi m_{3}\left(C_{1}+\frac{\Delta t}{\Delta x} C_{2}+\frac{\Delta t}{\Delta y} C_{3}\right) .
\end{aligned}
$$

